Les spectroscopistes le savent bien : plusieurs facteurs provoquent l'élargissement des raies de photoémission, ce qui réduit la précision des mesures. Parmi ces facteurs, certains sont liés aux performances des équipements utilisés, d'autres aux paramètres physiques de l'échantillon. C'est le cas de l'élargissement Doppler translationnel, connu et pris en compte lors de l'analyse des spectres.

En revanche, un autre effet Doppler, lié au mouvement de rotation des molécules, n'était jusqu'à présent pas pris en compte. II s'agit là d'un effet extrêmement faible par rapport aux énergies élevées des rayons $\mathrm{X}$.

Mais la résolution spectrale accessible sur la ligne de lumière PLEIADES est telle, qu'il a été possible pour la première fois au monde de le mettre clairement en évidence sur la molécule d'azote $\mathrm{N}_{2}$.

\title{
Comment suivre à la trace les mouvements des atomes dans les gaz?
}

Catalin Miron (catalin.miron@synchrotron-soleil.fr)

Synchrotron SOLEIL, L’Orme des Merisiers, Saint-Aubin, BP 48, 91192 Gif-sur-Yvette Cedex

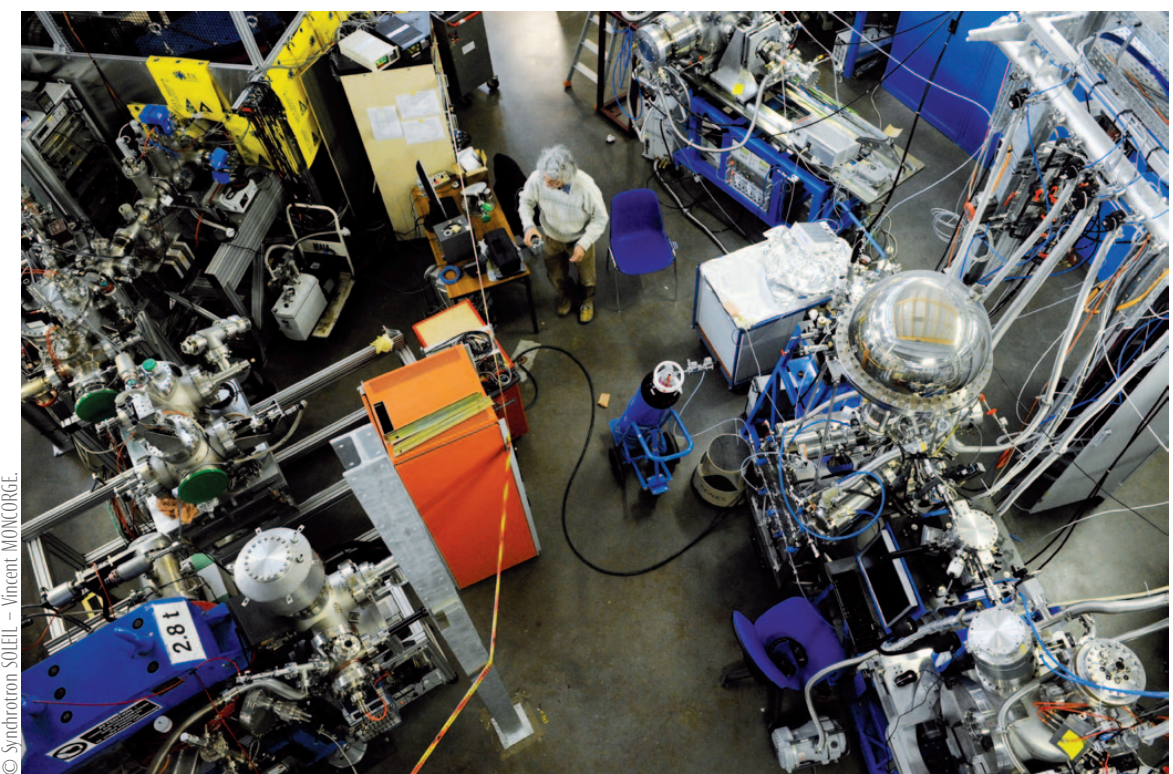

Vue du spectromètre d'électrons de la ligne PLEIADES, avec lequel les mesures ont été faites.

Une technique de pointe pour étudier la matière et l'analyser chimiquement

La spectroscopie d'électrons, ou de photoémission, consiste à extraire à l'aide d'un rayonnement électromagnétique de haute énergie (le plus souvent des rayons X) les électrons d'un atome, d'une molécule ou d'un matériau, et à en mesurer les paramètres physiques, comme leur énergie cinétique ou leur distribution angulaire dans le référentiel du laboratoire. L'intérêt de cette technique réside en un accès direct à l'une des propriétés fondamentales de la matière : sa structure électronique. Ce lien est fourni par la fameuse loi d'Einstein de l'effet photoélectrique, qui s'écrit pour un atome isolé : $E_{l}=h v-E_{c}$, où $h$ est la constante de Planck et $v$ la fréquence du rayonnement excitateur ; $E_{c}$ est l'énergie cinétique de l'électron émis et $E_{l}$ son énergie de liaison dans l'atome, qui caractérise les niveaux d'énergie et donc la structure électronique de l'échantillon.

Comme la structure électronique régit la nature et la force des liaisons chimiques entre les atomes, cette méthode d'analyse est très répandue, non seulement en physique, mais aussi en chimie et en science des matériaux. C'est Kai Siegbahn, professeur de physique à l'Université d'Uppsala et lauréat du Nobel de Physique en 1982, qui a établi la technique $\mathrm{ESCA}^{(\mathrm{a})}$ comme une méthode de choix pour l'analyse chimique, basée sur la grande spécificité des énergies de liaison des électrons en fonction de la nature des éléments et de leur environnement chimique immédiat. 


\section{\> \\ Améliorer notre connaissance de l'interaction rayonnement- matière}

Le domaine des rayons $\mathrm{X}$ mous, allant de 100 à 1500 électrons-volts (eV) environ, couvre les seuils d'ionisation en couches $\mathrm{K}$ et $\mathrm{L}$ des éléments légers des trois premières lignes du tableau périodique. Ces éléments sont extrêmement importants, car ils sont les constituants de base de la matière vivante $(\mathrm{C}, \mathrm{N}, \mathrm{O})$, mais aussi ceux de notre environnement, ou bien de matériaux à propriétés tout à fait exceptionnelles comme le graphène (voir l'article de A. Tejeda et al.).

Au-delà des aspects de caractérisation évoqués ci-dessus, l'étude des systèmes isolés, libres de toute interaction avec un quelconque environnement, permet d'aborder des questions aussi intéressantes et variées que les fascinants phénomènes de localisation dans des objets hautement symétriques, ou la photochimie sélective d'espèces complexes basée sur la «manipulation" d'électrons internes choisis. Ces études permettent d'améliorer considérablement notre connaissance des fondements de l'interaction rayonnement/ matière.

\section{La spectroscopie d'électrons et ses applications}

La mesure en laboratoire des spectres de photoémission se fait à l'aide de spectromètres d'électrons. Ces instruments, souvent très sophistiqués, sont optimisés pour obtenir une très haute résolution lors de l'analyse [1], ou pour leur efficacité géométrique de collection, en vue de réaliser des expériences de coïncidence [2] entre plusieurs particules issues du processus de photo-ionisation.

Lorsque c'est la très haute résolution qui est visée, une maitrise approfondie de toutes les causes possibles d'élargissement des raies de photoémission est impérative. Parmi celles-ci, il y a des causes dites homogènes, modélisées par une courbe lorentzienne (L), comme l'élargissement naturel des niveaux d'énergie associé à la durée de vie limitée des états ; mais aussi des causes dites inhomogènes, modélisées par une courbe gaussienne $(G)$. Ces dernières comprennent toutes les causes instrumentales (la largeur spectrale du rayonnement électromagnétique excitateur, mais aussi la résolution des spectromètres utilisés), ainsi que les causes intrinsèques à l'échantillon, comme l'élargissement Doppler (voir encadré 1) des spectres, dû à l'agitation thermique des molécules.

\section{À température ambiante, les atomes et les molécules bougent}

L'effet Doppler translationnel, lié au mouvement de translation des espèces isolées en phase gazeuse, est connu et pris en compte depuis des décennies par les spectroscopistes lors de l'analyse de leurs résultats. Ce n'était par contre pas le cas d'un autre effet Doppler, lié au mouvement de rotation des molécules (fig. 1). Comme illustré dans la figure 1, ce mouvement de rotation va affecter, de façon similaire à celui de translation, l'énergie cinétique des électrons émis, en induisant un élargissement des raies de photoémission. Il s'agit ici d'un effet purement moléculaire, qui n'a pas lieu d'être pour des échantillons atomiques, les atomes n'ayant pas de moment d'inertie associé comme les molécules. Cet effet, prédit initialement par un groupe de théoriciens suédois de l'Institut royal de technologie de Stockholm [3], est extrêmement faible par rapport aux énergies élevées mises en jeu par les rayons X. Cependant, la résolution instrumentale accessible sur la ligne de lumière PLEIADES [4] est telle qu'il a été possible, pour la première fois au monde, de mettre clairement en évidence cet élargissement Doppler rotationnel sur la molécule d'azote $\mathrm{N}_{2}$ [5-8].

\section{L'effet Doppler}

L'effet Doppler est le décalage de fréquence d'une onde acoustique ou électromagnétique entre la mesure à l'émission et la mesure à la réception, lorsque la distance entre l'émetteur et le récepteur varie au cours du temps.

L'effet Doppler est un phénomène dont le nom fait partie du langage courant, notamment pour ses manifestations acoustiques : la fréquence de l'onde sonore émise par un objet en mouvement - un véhicule, par exemple - et perçue par un observateur, sera différente selon la vitesse et la direction du mouvement de cet objet. Toujours dans le cas d'ondes acoustiques, ce phénomène sert, en médecine par exemple, à mesurer la vitesse du sang. À une échelle de taille nettement supérieure, il est utilisé pour mesurer la vitesse des corps célestes (par analyse de la lumière qu'ils émettent).

Or, les atomes et les molécules en phase gazeuse se déplacent également. Comme pour la lumière émise par les étoiles, la valeur de l'énergie cinétique des électrons émis - mesurée par spectroscopie de photoélectrons - sera différente selon la vitesse et la direction de déplacement des atomes ou des molécules qui les émettent. En phase gazeuse, le mouvement thermique des molécules est aléatoire et mène donc à un élargissement des pics, appelé élargissement Doppler translationnel.
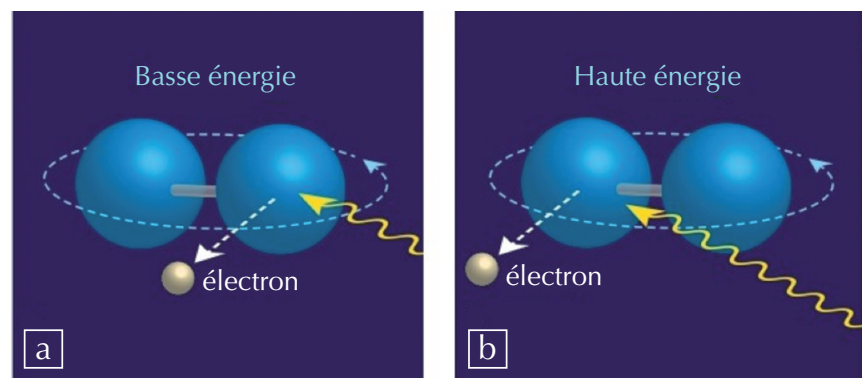

1. Représentation schématique du mécanisme de l'effet d'élargissement Doppler rotationnel sur une molécule diatomique mononucléaire.

Si l'électron est considéré émis de façon localisée à partir d'un seul des deux atomes dans la direction opposée à la rotation moléculaire, son énergie cinétique se verra diminuée (a), alors que s'il est émis dans la même direction que celle de la rotation moléculaire, son énergie cinétique se verra augmentée (b), ce qui se manifestera in fine par un élargissement.

(Figure reproduite à partir de la publication associée parue dans American Scientist - O B Barbara Aulicino/American Scientist). 
Cet élargissement est facilement décelable sur la figure 2, qui montre le spectre de photoémission d'un échantillon constitué d'un mélange d'azote $\left(\mathrm{N}_{2}\right)$ et de krypton $(\mathrm{Kr})$. Les raies de photoémission du krypton ne sont affectées que par les causes instrumentales (les mêmes pour tous les pics) et par l'effet Doppler translationnel, dépendant uniquement de l'énergie cinétique de l'électron et de la masse de l'émetteur. Les raies moléculaires sont, elles, affectées en plus par l'élargissement Doppler rotationnel, qui les rend plus larges que les raies atomiques.

Ce travail, réalisé en collaboration avec des équipes des universités de l'Oregon et de New York, de Turku et de Sendai, est une petite révolution dans le monde des spectroscopistes, et indique que désormais toute mesure de spectroscopie de photoémission à haute résolution doit prendre en considération cet effet. Ces résultats ont été publiés dans $P R L[5]$ et repris par différents médias français [6] et internationaux $[7,8]$.

Cependant, il est important de noter qu'il ne s'agit pas ici uniquement d'un très bel exploit expérimental, qui permettra de modéliser plus précisément les spectres d'électrons à très haute résolution. Contrairement à l'élargissement translationnel, l'élargissement Doppler rotationnel a une dépendance angulaire.
En effet, à partir de la figure 1a, il est facile de comprendre que si l'électron est émis de façon préférentielle le long de l'axe internucléaire, il n'y aura pas d'élargissement Doppler rotationnel, car l'énergie cinétique de l'électron ne sera pas influencée par la rotation de la molécule. A contrario, l'élargissement Doppler rotationnel sera maximum si l'électron est émis perpendiculairement à l'axe internucléaire, comme dans la situation illustrée sur la figure $1 \mathrm{~b}$.

C'est précisément de cette manière que la magnitude de l'élargissement Doppler rotationnel contient des informations sur la distribution angulaire des électrons dans le référentiel propre de la molécule. Or, la distribution angulaire des électrons est un des paramètres clés caractérisant le phénomène de photo-ionisation, et une signature de la symétrie des états électroniques. Ainsi, les légères différences de largeur observées sur la figure 2 entre les raies associées aux différents états électroniques $\mathrm{X}, \mathrm{A}$ et $\mathrm{B}$, reflètent leurs différentes symétries. Cette dépendance angulaire est discutée dans le cadre de la modélisation théorique de l'effet, proposée dans la référence [5]. De surcroît, de nouveaux travaux sont en cours, qui explorent plus en détail la capacité prédictive des mesures de ce type pour la caractérisation des symétries des états.

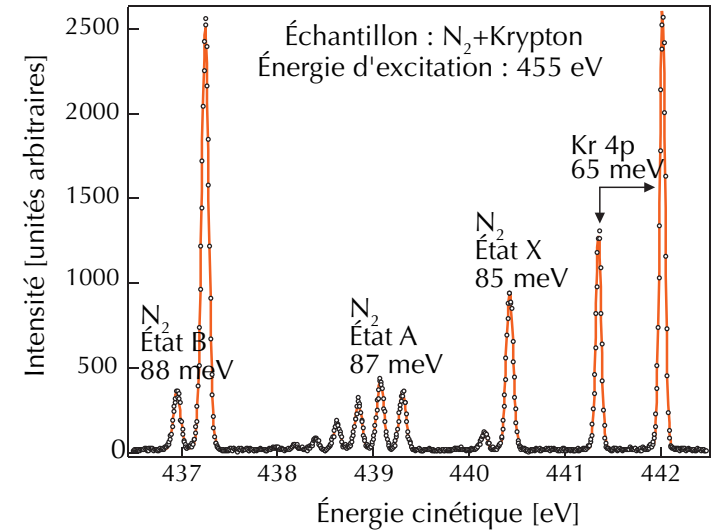

2. Spectre de photoémission enregistré sur la ligne de lumière PLEIADES du synchrotron SOLEIL pour un mélange d'azote et de krypton, à une énergie excitative de $455 \mathrm{eV}$. Alors que les deux raies (composantes spin-orbite) du krypton ne sont affectées que par les élargissements instrumentaux et Doppler translationnel, les raies moléculaires de l'azote sont affectées de surcroît par l'effet d'élargissement Doppler rotationnel. Dans le cas présent, ce dernier est significatif, de l'ordre de $40 \mathrm{meV}$, et tout à fait comparable à l'élargissement Doppler translationnel (environ 49 meV). Les raies moléculaires correspondent à l'état fondamental $(X)$ et aux deux premiers états excités ( $\mathrm{A}$ et $\mathrm{B}$ ) de l'ion $\mathrm{N}_{2}{ }^{+}$, produits par photo-ionisation en couche de valence. Chacun de ces états électroniques moléculaires présente une structure fine, décrivant les différents états vibrationnels excités dans le processus de photo-ionisation. Les largeurs instrumentales indiquées sur la figure (p.e. $85 \mathrm{meV}$ pour l'état $\mathrm{X}$ de $\mathrm{N}_{2}$ ) sont mesurées à mi-hauteur des raies individuelles, et elles sont toutes les mêmes pour un même état électronique.
La ligne

de lumière PLEIADES

PLEIADES (Polarized Light source for Electron and Ion Analysis from Diluted Excited Species) est une ligne de lumière de rayons $\mathrm{X}$ mous à ultra-haute résolution spectrale (pouvoir résolvant ultime avoisinant les 100000 à $50 \mathrm{eV}$ ), couvrant le domaine d'énergie entre $7 \mathrm{eV}$ et $1 \mathrm{keV}$.

Cet instrument est dédié aux études spectroscopiques de physique atomique et moléculaire. Tous les états de polarisation horizontale, verticale et linéaire tournante, ainsi qu'elliptique, sont disponibles à partir de $55 \mathrm{eV}$, grâce à deux onduleurs magnétiques.

L'ultra-haute résolution spectrale fait de PLEIADES une ligne unique au monde. Elle est obtenue grâce à un monochromateur original conçu à SOLEIL. Ce monochromateur emploie des réseaux de diffraction plans, focalisants, et à profondeur de gravure variable, ce qui permet d'optimiser leur efficacité, quelle que soit la longueur d'onde sélectionnée.

Trois montages expérimentaux sont installés : un spectromètre d'électrons à haute résolution, un montage de coïncidences entre des électrons Auger et des ions résolus en énergie et en angle (EPICEA), ainsi qu'un montage (MAIA) permettant les études de photoionisation d'ions positifs et négatifs.

(a) ESCA : "Electron Spectroscopy applied to Chemical Analysis".

\section{Références}

1 C. Miron et P. Morin, dans Handbook of high-resolution spectroscopy, Éditeurs : F. Merkt et M. Quack, John Wiley and Sons, Inc., pp. 1655-1689 (2011).

2 $\bullet$ C. Miron et P. Morin, Nucl. Instrum. Methods Phys. Res., Sect. A 601 (2009) 66.

3• Y.-P. Sun et al., Phys. Rev. A 82 (2010) 052506.

$4 \bullet$ www.synchrotron-soleil.fr/Recherche/Lignes Lumiere/PLEIADES

5• T.D. Thomas et al., Phys. Rev. Lett. 106 (2011) 193009

6• www.futura-sciences.com/fr/news/t/physique1/d/leffet-doppler-rotationnel-moleculaire-enfinobserve_30134/

7• "Tumbling of Molecules Affects Experiments", Physical Review Focus, 27, Story 19 (2011) http://focus.aps.org/story/v27/st19

8• "Minuscule Speed Traps: Rotating molecules experience the Doppler effect", American Scientist, 99 (2011) 300. www.americanscientist.org/issues/pub/minuscule-speed-traps/1 